\title{
Development of A Robust and Scalable Process for the Large-scale Preparation of Vadadustat
}

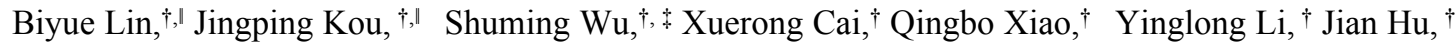
Jianbing Li, ${ }^{\dagger}$ Zhongqing Wang ${ }^{*}, \dagger,+$,

${ }^{\dagger}$ Department of Process Research and Development, HEC Pharm Group, Dongguan 523871, P. R. China

*State Key Laboratory of Anti-Infective Drug Development (NO. 2015DQ780357), Sunshine Lake Pharma Co., Ltd, Dongguan 523871, P. R. China

${ }^{\S}$ School of Pharmacy, Xiangnan University, Chenzhou 423000 Hunan, China

*E-mail:Wangzhongqing@hec.cn

\section{Supporting Information}

Table of Contents

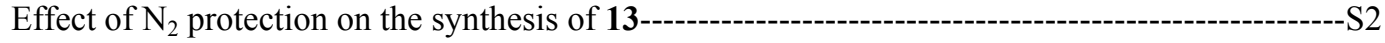

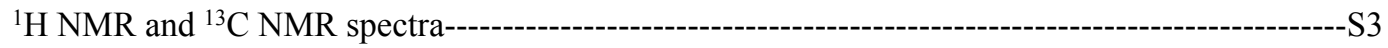

HPLC spectra of Vadadustat --- 
Table S1. Effect of $\mathrm{N}_{2}$ protection on the synthesis of $\mathbf{1 3}^{\mathrm{a}}$

\begin{tabular}{cccccc}
\hline \multirow{2}{*}{ entry } & Approaches & Time $(\mathrm{h})$ & \multicolumn{3}{c}{ HPLC (\%) } \\
\cline { 4 - 6 } 1 & & & $\mathbf{1 0}$ & $\mathbf{2 0}$ & $\mathbf{1 3}$ \\
\hline 2 & $\mathrm{~N}_{2}$ protection & $5 \mathrm{~h}$ & 6.40 & 0.49 & 88.33 \\
3 & & $11 \mathrm{~h}$ & 2.72 & 0.52 & 93.24 \\
4 & & $21 \mathrm{~h}$ & 1.10 & 0.79 & 93.20 \\
\hline 5 & No protection & $5 \mathrm{~h}$ & 8.14 & 0.34 & 88.36 \\
6 & & $11 \mathrm{~h}$ & 3.94 & 0.58 & 91.96 \\
\hline
\end{tabular}

a reaction conditions: 1) 10 (1.0 g), NaOMe (30\% in $\mathrm{MeOH}, 2.0$ equiv), methanol (MeOH, $10.0 \mathrm{~mL})$, reflux for $6.0 \mathrm{~h}$; 2) water $(10.0 \mathrm{~mL}), \mathrm{NaOH}(2.5$ equiv), reflux for $6.0 \mathrm{~h}$ 


\section{${ }^{1} \mathrm{H}$ NMR and ${ }^{13} \mathrm{C}$ NMR spectra}

${ }^{1} \mathrm{H}$ NMR and ${ }^{13} \mathrm{C}$ NMR spectra for compound 10
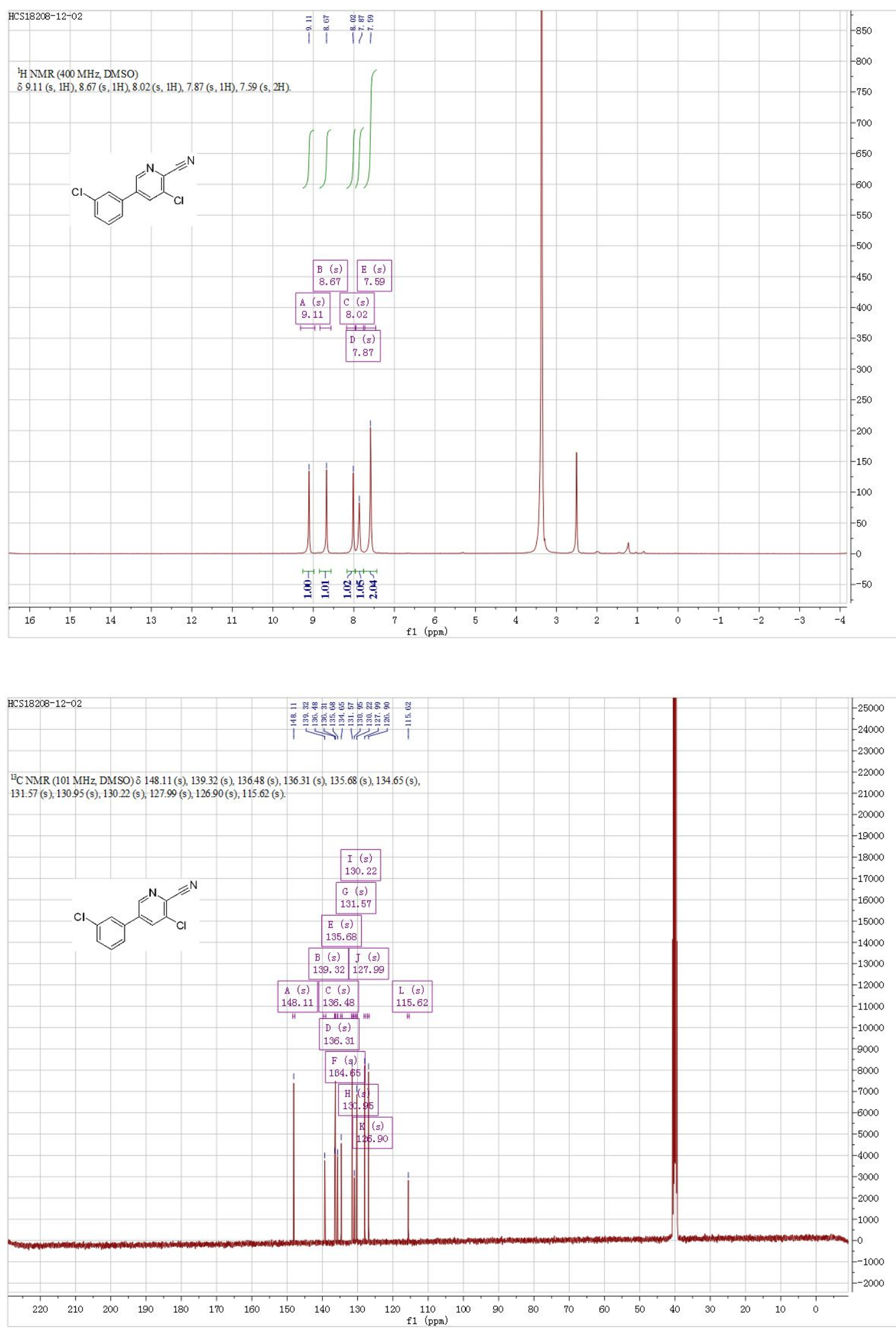
${ }^{1} \mathrm{H}$ NMR and ${ }^{13} \mathrm{C}$ NMR spectra for compound 13
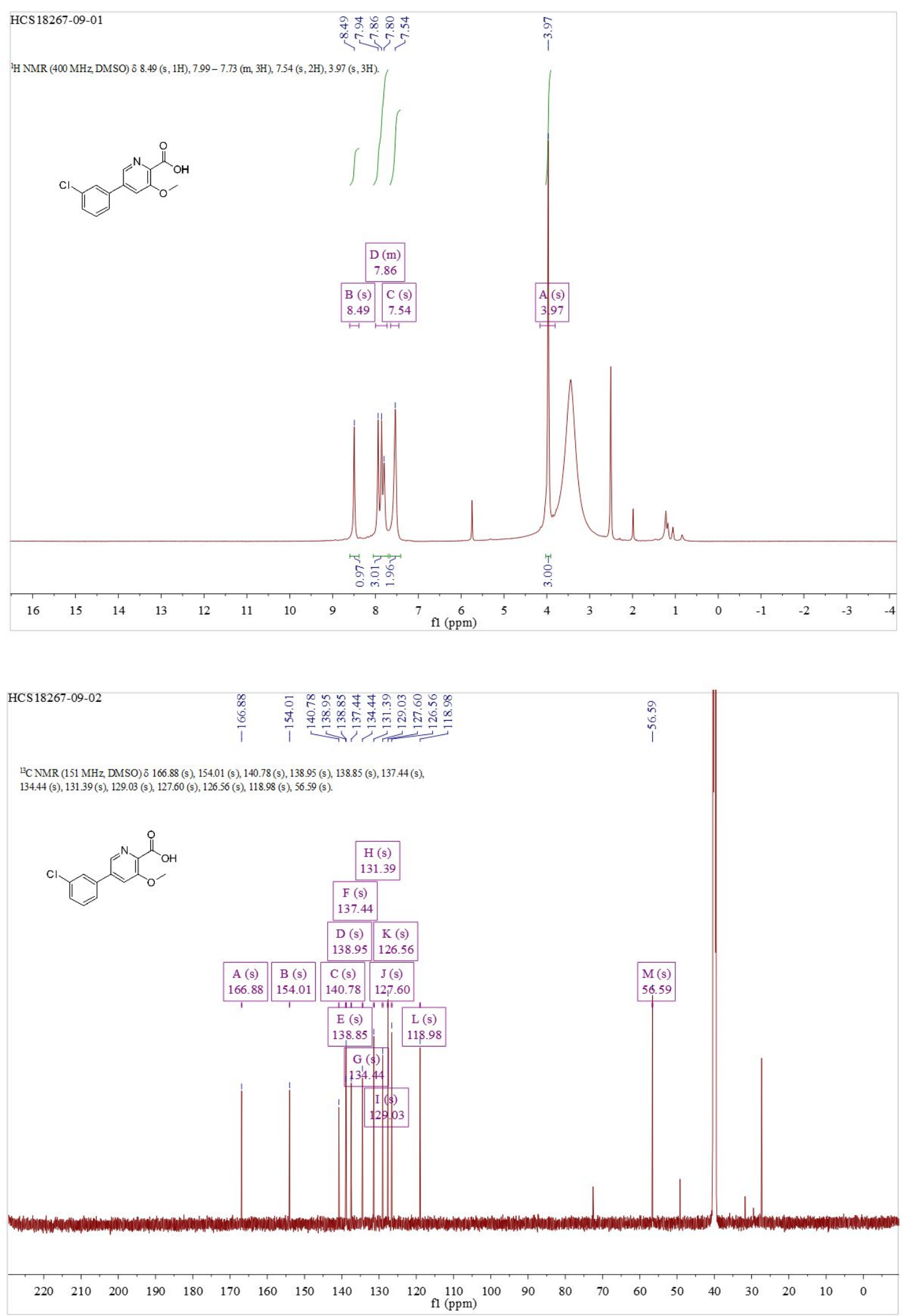
${ }^{1} \mathrm{H}$ NMR and ${ }^{13} \mathrm{C}$ NMR spectra for compound 14
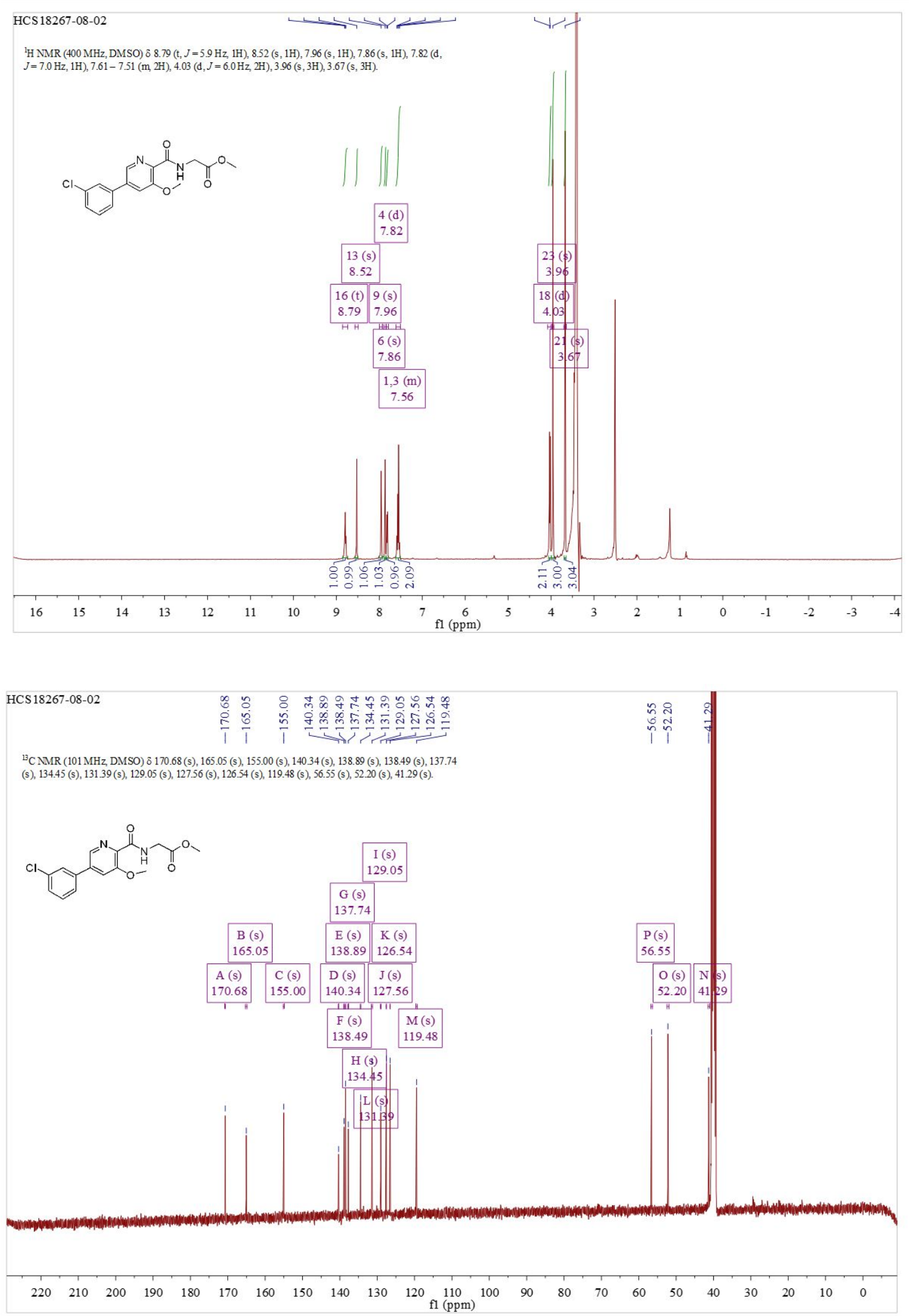
${ }^{1} \mathrm{H}$ NMR and ${ }^{13} \mathrm{C}$ NMR spectra for vadadustat
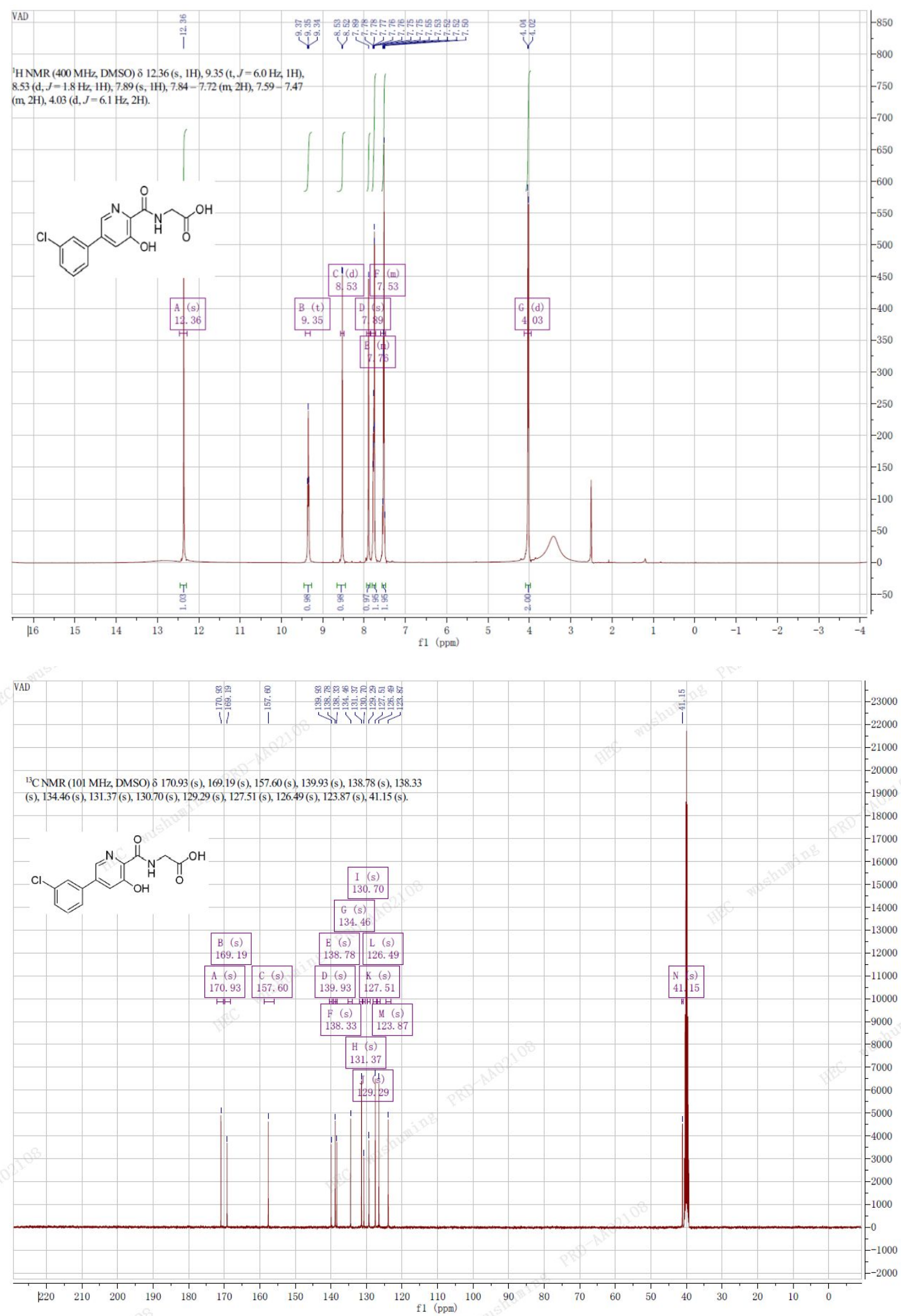
HPLC spectra of vadadustat

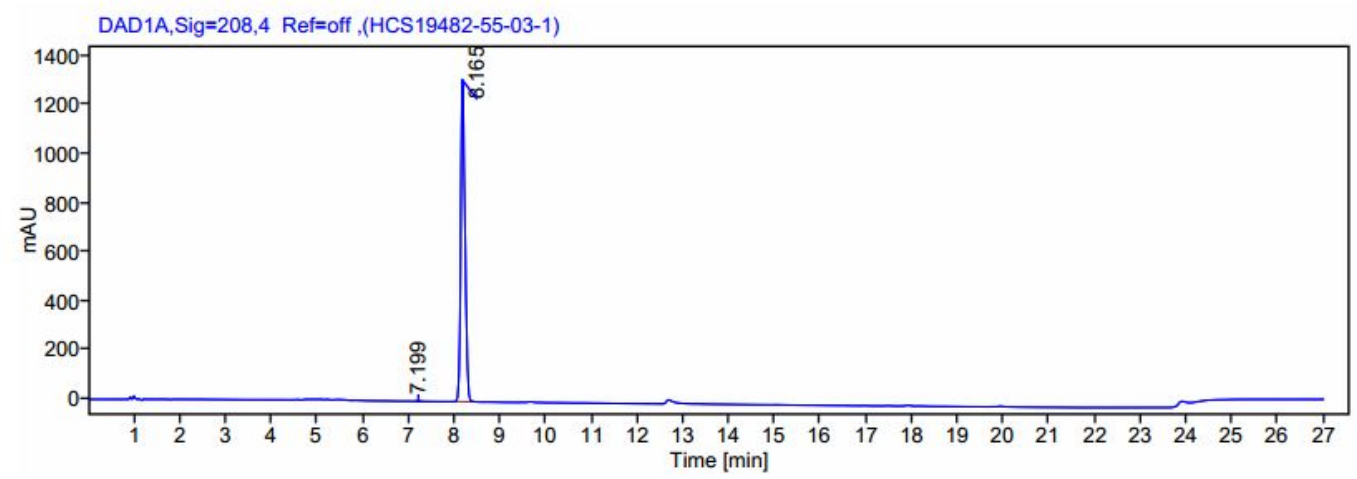

Signal: $\quad$ DAD1A,Sig $=208,4$ Ref $=$ off

$\begin{array}{ccccccc}\text { RT [min] } & \text { Height } & \text { Area } & \text { Area \% } & \begin{array}{c}\text { Resolut } \\ \text { ion USP }\end{array} & \text { Tail } & \begin{array}{c}\text { Theoretical } \\ \text { Plates USP }\end{array} \\ 7.20 & 1.316 & 5.838 & 0.07 & & 0.9 & 58113 \\ 8.17 & 1310.947 & 8418.053 & 99.93 & 6.6 & 1.2 & 36494\end{array}$

